\title{
Thickness dependence of the properties of indium tin oxide (ITO) FILMS prepared by activated reactive evaporation
}

\author{
M.D. Benoy \\ Department of physics, M.A. College, Kothamangalam, Kerala, India - 686666 \\ E.M. Mohammed, ${ }^{*}$ Suresh Babu M., and Binu P.J. \\ Department of Physics, Maharaja's College, Ernakulam, Kerala, India - 682011 \\ B. Pradeep \\ Department of Physics, Cochin University of Science and Technology, Kochi Kerala, India - 682022
}

(Received on 14 Januarry, 2009)

\begin{abstract}
Tin doped indium oxide thin films were prepared on glass substrates kept at room temperature, by activated reactive evaporation (ARE). Structural, electrical and optical properties were studied for films having different thickness. The resulting films are polycrystalline and show $\approx 90 \%$ transmission in the visible region. Hall effect measurements at room temperature for a film with a nominal thickness of $\approx 350 \mathrm{~nm}$ shows a relatively high carrier concentration $\approx 6.3 \times 10^{20} \mathrm{~cm}^{-3}$, mobility $\approx 16 \mathrm{~cm}^{2} V^{-1} \mathrm{~s}^{-1}$, with a low resistivity $\approx 1.01 \times 10^{-3} \Omega \mathrm{cm}$.
\end{abstract}

Keywords: Thin films, ITO, ARE

\section{INTRODUCTION}

The interest in transparent conductors has tremendously increased, since the first report of a transparent conducting cadmium oxide films by Badeker(1907) [1]. These materials find applications in liquid crystal displays, transparent electrodes for solar cells, gas sensors, photodetectors and laser damage resistant coatings in high power laser technology [2]. The simultaneous occurrence of high optical transparency $(\approx 90 \%)$ in the visible region together with high electrical conductivity require the creation of electron degeneracy in wide band gap ( $\geq 3 \mathrm{eV}$ ) oxides by introducing nonstoichiometry or appropriate dopants. These conditions can be achieved in various oxides of indium, tin, cadmium, zinc and in combinations thereof.

In view of the technological importance, a great deal of basic research and development has been carried out on the electrical and optical properties of indium oxide based materials. Rupprecht [3] carried out one of the first investigations on the indium oxide $\left(\mathrm{In}_{2} \mathrm{O}_{3}\right)$. Since then different techniques have been used for the preparation of these oxide films [4-6]. The conductivity of these films can be increased by introducing various type of dopants such as tin, cadmium, antimony and fluorine. Considerable attention has been given to the study of tin doped indium oxide (ITO) films, because of their enhanced electrical and optical quality, compared to other transparent conductors. ITO is an $\mathrm{In}_{2} \mathrm{O}_{3}$ based material that has been doped with $\mathrm{Sn}$ to improve the electrical conductivity. Here tin acts as a cationic dopant in the $\mathrm{In}_{2} \mathrm{O}_{3}$ lattice and substitute on the indium sites to bind with the interstitial oxygen. The ITO films, with a band gap greater than 3 $\mathrm{eV}$ is highly transmitting in the visible region and hence is preferred in most of the applications.

In the present paper we report on the thickness dependence of electrical and optical properties of ITO films, prepared by activated reactive evaporation.

*Electronic address: emmohammed_2005@yahoo.com

\section{EXPERIMENTAL}

Indium tin oxide films were prepared on substrates kept at room temperature by activated reactive evaporation [7]. The starting materials were In and $10 \mathrm{Wt} \% \mathrm{Sn}$. The purity of each metal was $99.999 \%$. A resistively heated molybdenum boat was used to evaporate the material, in a conventional vacuum system. The evaporation was carried out in the presence of oxygen plasma. The vacuum chamber was first evacuated to a pressure of $10^{-5} \mathrm{~m}$.bar. Then industrial grade oxygen was admitted into the chamber, through a needle valve, to a pressure of $5 \times 10^{-3} \mathrm{~m}$.bar. The anode supply was turned on, initiating the glow discharge. A bluish glow filled the whole chamber, and a steady discharge current was maintained. The indium and tin placed in the molybdenum boat was evaporated in the oxygen plasma. A slight adjustment of the needle valve was necessary to maintain the oxygen pressure at $5 \times 10^{-3} \mathrm{~m}$.bar. Optically flat glass slides were used as substrates. Substrates were cleaned with an industrial detergent, followed by running water and ten minutes ultrasonic agitation in distilled water. The substrates were dried in hot air and loaded into the chamber. The details of the deposition parameters have been reported in our previous paper [8].

The crystallinity of the films were identified using X-ray diffraction. The thickness of the films were measured using Tolansky's multiple beam interferometric method [9]. Conductivity and Hall effect measurements were carried out using van der Pauw method. Samples used for Hall measurements were $1 \mathrm{~cm} \times 1 \mathrm{~cm}$ in size and the ohmic contacts were made using silver paste. The transmission spectra were recorded from $2600 \mathrm{~nm}$ to cut off using Hitachi U-3410 UVVis - NIR spectrophotometer.

\section{RESULTS AND DISCUSSIONS}

Figure 1 shows the X-ray diffraction pattern of ITO films having various thicknesses. Figure 1(a) shows the XRD pattern of film with thickness $\approx 80 \mathrm{~nm}$. It gives reflections only 


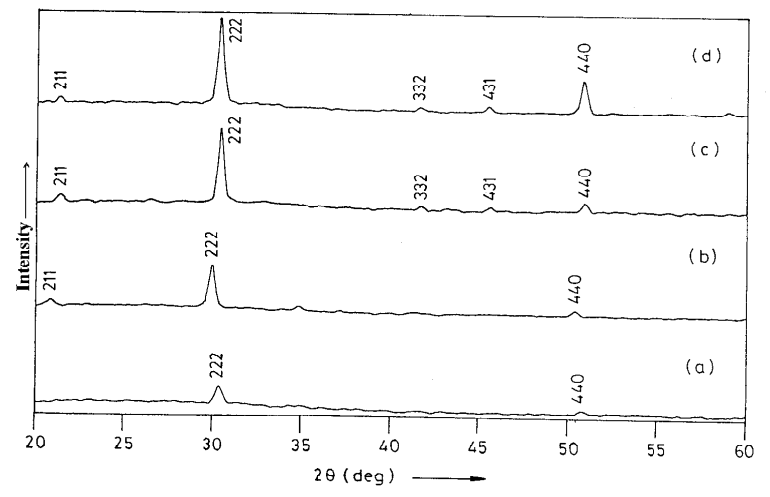

FIG. 1: X-ray diffraction pattern of ITO films of different thickness: (a) $80 \mathrm{~nm}$; (b) $150 \mathrm{~nm}$; (c ) $270 \mathrm{~nm}$; (d) $350 \mathrm{~nm}$

TABLE 1: X-ray diffraction data of ITO films of different thickness with the standard data

\begin{tabular}{|c|c|c|c|c|c|c|c|c|c|c|}
\hline \multirow{2}{*}{\multicolumn{3}{|c|}{$\begin{array}{c}\text { Standard data } \\
\text { JCPDS File No.6-416 }\end{array}$}} & \multicolumn{8}{|c|}{ Film thickness } \\
\hline & & & \multicolumn{2}{|c|}{$80 \mathrm{~nm}$} & \multicolumn{2}{|c|}{$150 \mathrm{~nm}$} & \multicolumn{2}{|c|}{$270 \mathrm{~nm}$} & \multicolumn{2}{|c|}{$350 \mathrm{~nm}$} \\
\hline (hkl) & $\mathrm{dA}^{0}$ & $\mathrm{I} / \mathrm{I}_{0}$ & $\mathrm{~d} \mathrm{~A}^{0}$ & I (cps) & $\mathrm{d} \mathrm{A}^{0}$ & I (cps) & $\mathrm{d} \mathrm{A}^{0}$ & I (cps) & $\mathrm{dA}^{0}$ & I (cps) \\
\hline (211) & 4.13 & 14 & ---- & ---- & 4.125 & 185 & 4.125 & 349 & 4.125 & 200 \\
\hline (222) & 2.92 & 100 & 2.91 & 336 & 2.91 & 849 & 2.91 & 1539 & 2.91 & 2023 \\
\hline (332) & 2.157 & 6 & --- & ---- & ---- & ---- & 2.155 & 173 & 2.155 & 100 \\
\hline (431) & 1.984 & 10 & ---- & ---- & ---- & --- & 1.981 & 120 & 1.981 & 140 \\
\hline (440) & 1.788 & 35 & 1.786 & 195 & 1.786 & 106 & 1.786 & 275 & 1.786 & 856 \\
\hline
\end{tabular}

from (222) and (440) planes, which are having relative intensity 100 and 35 respectively in the standard XRD data (JCPDS File No. 6- 416). Figure1(b) shows the XRD pattern for a film with thickness $\approx 150 \mathrm{~nm}$, which gives reflection from (211) plane also, which is having a relative intensity 14 in the standard data, in addition to the (222) and (440) planes. Figure 1 (c and d) shows the XRD pattern of films having thickness 270 and $350 \mathrm{~nm}$ respectively. Here reflections from (332) and (431) planes with relative intensity 6 and 10 get resolved. It shows that as the film thickness increases the XRD pattern agrees very well with the standard data. In the case of film with smaller thickness, only those planes having a higher relative intensity is resolved.

It is a well-known fact that the reflected X-ray intensity depends on the penetration depth of X-ray. For films with thickness $\approx 80 \mathrm{~nm}$, we obtained only the reflections corresponding to the high relative intensity in the standard data. Still the total count was very small compared to films with higher thickness. Results of XRD analysis were summarised in table 1. From this it is very clear that the films formed at room temperature are polycrystalline in nature.

The absence of reflections from (211), (322) and (431) plains of the films, with smaller thickness are not due to orientation, but due to the lack of sample thickness. This is evident by the comparative studies given in table1. The lattice parameter is calculated to be $10.17 A^{0}$, which is very close to that of $\mathrm{In}_{2} \mathrm{O}_{3}$.

The grain size was calculated from the XRD pattern using Debye-Scherrer formula[10]. Figure 2 shows the variation of grain size with thickness. It can be seen that the grain size was independent of thickness, for films with thickness greater than $150 \mathrm{~nm}$. Here the average grain size is $\approx 16 \mathrm{~nm}$. Figure 3 shows the variation of the sheet resistance with the

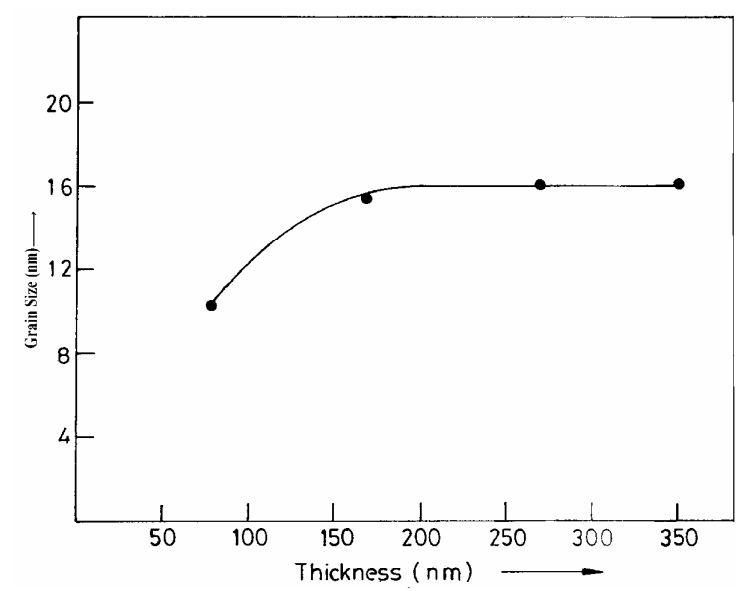

FIG. 2: Variation of the grain size with film thickness (d).

film thickness.

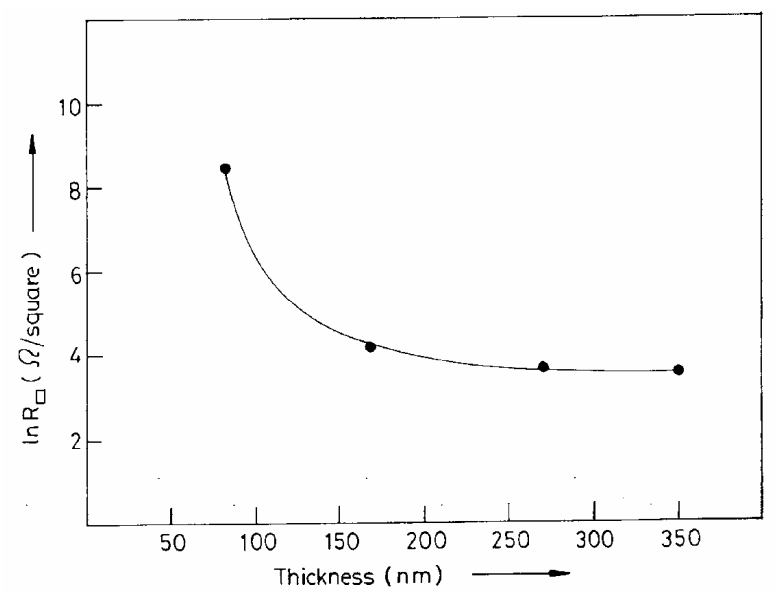

FIG. 3: Variation of sheet resistance (R) with the film thickness.

It was observed that the decrease in sheet resistance is rapid when the film thickness increases from $80 \mathrm{~nm}$ to 150 $\mathrm{nm}$, whereas this decrease is very slow when the thickness is greater than $150 \mathrm{~nm}$. A similar variation in sheet resistance with thickness has been reported for ITO and $\mathrm{SnO}_{2}$ films $[11,12]$. The observed increase in sheet resistance for films having thickness less than $150 \mathrm{~nm}$ is probably due to the discontinuous nature of the film. The observed dependence of sheet resistance on thickness is in good agreement with Fuchs-Sondheimer theory [13].

Figure 4 show the transmission spectra of ITO films with different thickness. The transmittance in the visible region is found to be almost independent of film thicknesses. But the transmission in the NIR region decreases with increase in film thickness.

Figure 5 shows the reflection spectra of the same set of films. It shows that there is no appreciable reflection in the NIR region. But in case of samples having grater thickness, the reflectance increases slightly for wavelengths above 2000 


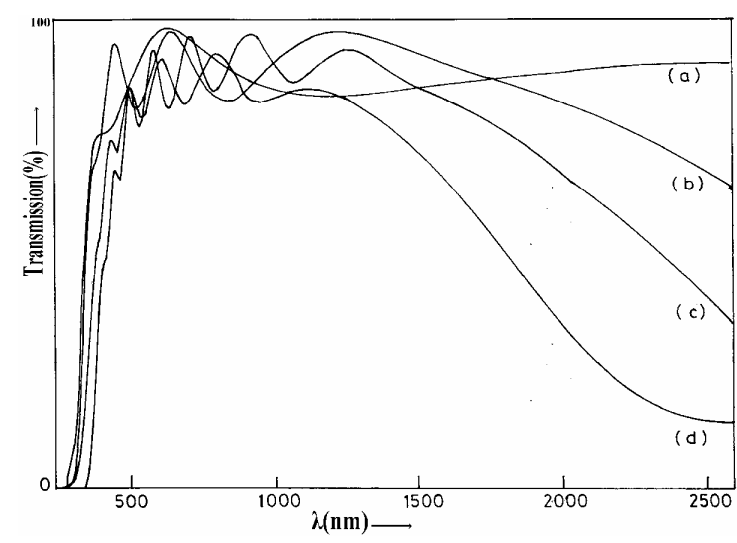

FIG. 4: Transmission spectra of ITO films of different thickness: (a) $80 \mathrm{~nm}$; (b) $150 \mathrm{~nm}$; (c ) $270 \mathrm{~nm}$; (d) $350 \mathrm{~nm}$.

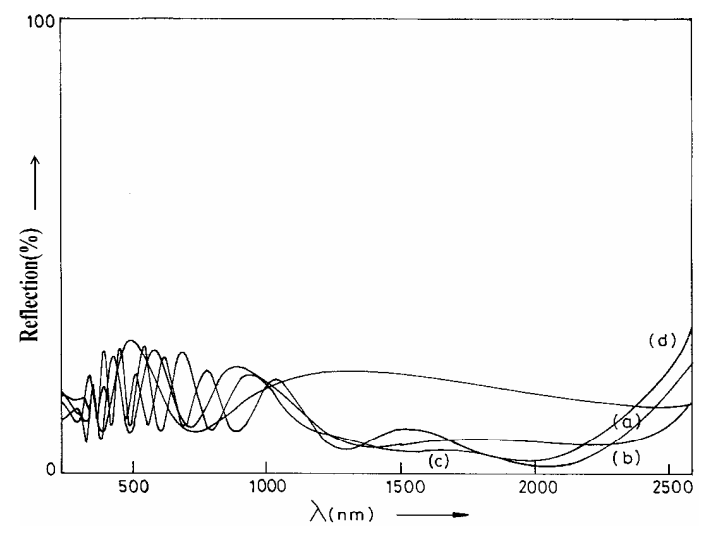

FIG. 5: Reflection spectra of ITO films of different thickness; (a) $80 \mathrm{~nm}$;(b) $150 \mathrm{~nm}$; (c ) $270 \mathrm{~nm}$; (d) $350 \mathrm{~nm}$.

TABLE 2: Data obtained from Hall effect measurement of ITO films at room temperature.

\begin{tabular}{|c|c|c|c|}
\hline $\mathrm{d}(\mathrm{nm})$ & $\mathrm{n}\left(\mathrm{cm}^{-3}\right)$ & $\rho(\Omega-\mathrm{cm})$ & $\mu\left(\mathrm{cm}^{2} \mathrm{~V}^{-1} \mathrm{~s}^{-1}\right)$ \\
\hline 80 & $3.87 \times 10^{18}$ & $3.3 \times 10^{-2}$ & 48 \\
\hline 150 & $1.12 \times 10^{20}$ & $1.4 \times 10^{-3}$ & 34 \\
\hline 270 & $3.8 \times 10^{20}$ & $1.05 \times 10^{-3}$ & 28 \\
\hline 350 & $6.3 \times 10^{20}$ & $1.01 \times 10^{-3}$ & 16 \\
\hline
\end{tabular}

$\mathrm{nm}$. But this increase in reflectance is not consistent with the decrease in transmittance in the same region. So the decrease in transmittance with increase in thickness in the NIR region is due to the free carrier absorption, a phenomenon that is common in all transparent conductors having high carrier concentration [14].

It is also supported by the Hall effect studies. Table 2 show the results obtained from the Hall effect measurements. It shows that the carrier concentration increases with increase in film thickness. A similar variation in the carrier density with film thickness has been reported in the case of Sb doped $\mathrm{SnO}_{2}$ films by Kaneko and Miyake [15]. The decrease in transmittance in the NIR region with increase in thickness for ITO films has been observed by other authors also[11,13,16].

\section{CONCLUSION:}

Highly conducting and transparent ITO films were prepared on glass substrates at room temperature by activated reactive evaporation. Crystallinity and grain size of the films were found to increase with film thickness. The Hall effect measurement shows that for a given set of deposition conditions, the thicker films have better electrical properties. The visible transmittance was found to be independent of thickness, whereas the transmittance in the NIR region decreases with film thickness.

\section{Acknowledgments}

M.D. Benoy and E.M. Mohammed acknowledges University Grants Commission, Government of India for the financial assistance received for their projects.
[1] Badeker K, Ann. Phys. (Leipzig) 22749 (1907)

[2] Chopra K L, Major S and Pandya D K, Thin Solid Films 102 1(1983)

[3] Rupprecht G Z. Phys. 139 504(1954)

[4] Cruz L R O and Santos O J, Materials Letters 12 72(1991)

[5] Hongbin Ma, Jung-Soo Cho and Chung-Hoo Park, Surf. and Coatings Tech. 153 131(2002)

[6] Hirokazu Izumi, Fredirick O.A, Takahiro Kaneyoshi, Tsuguo Ishihara, Hideki Yoshika and Muneyuki Motoyama, J. Appl. Phys. 91 1213(2002)
[7] George J, Pradeep B and Joseph K S, Rev. Sci. Instrum. 57 2355(1986)

[8] Benoy M D and Pradeep B, Bull. of Mater. Sci. 20 1029(1997)

[9] Tolansky S., Multiple Beam Interferometry of Surface and Films (Oxford Univ. press, London) (1948)

[10] Cullity B D, Elements of X-ray Diffraction (Addison-Wesley, London) p.284(1978)

[11] Banerjee R, Ray S and Barua A K J.Mater.Sci.Lett. 6 1203(1987)

[12] Omar O A, Rigaie H F and Fikry W. F 
J.Mater.Sci.Mater.Electron. 379 [(1990)]

[13] Adurodija F O, Izumi H, Ishihara T, Yoshioka H., Yamada K, Ali M S, Khan K A and Khan M. S R, Phys. Stat. Sol (a). 149 611(1995)

[14] Mizuhashi M, Thin Solid Films 70 91(1980)
[15] Kaneko H and Miyake K, J.Appl.Phys. 53 362(1982)

[16] Li-jian Meng and dos Santos M. P, Thin Solid Films 303151 (1997) 